\title{
Application of Mathematical Modeling in Survival Time Prediction for Females with Advanced Cervical Cancer Treated Radio-chemotherapy
}

\author{
Jerzy A. Moczko ${ }^{1}$, Andrzej Roszak² \\ ${ }^{1}$ Chair and Department of Computer Science and Statistics \\ University of Medical Sciences in Poznań, Dabrowskiego 79, 60-529 Poznań, Poland \\ e-mail:jmoczko@am.poznan.pl \\ ${ }^{2}$ Wielkopolska Cancer Center, Garbary 15, 61-866 Poznań, Poland \\ e-mail:andrzej.roszak@wco.pl
}

(Rec. 11 July 2005)

\begin{abstract}
Clinical investigations yield huge amount of experimental data which need appropriate processing. High degree of their variability decreases quality of prognostic and classification results. Frequently problem of strong redundancy of variables incorporated into model occurs, produces computational instabilities and in multifactorial models makes the appropriate interpretation of obtained results hindered. In this paper we present the set of mathematical procedures used in comparative analysis of two treatment methods for patients with advanced cervical cancer: radiotherapy and combined treatment of ionizing irradiation and chemotherapy.
\end{abstract}

Keywords: survival analysis, mathematical modeling, cervical cancer, radio-chemotherapy

\section{INTRODUCTION}

In Poland, the number of female patients with cervical cancer has been observed for the past few decades to be stable with no improvements in the outcome of treatments. A program for detecting early stages of cervical cancer does not exist in Poland, resulting in a negative impact on the clinical stages of this cancer at the time of detection with most patients at stages II-IV. This has led to a poor treatment outcome placing Poland in the 5 year survival rate of cervical cancer patients at almost the last position among European countries (51\% of 5 year survival rate) [1].

Radiotherapy is used as the basic treatment of advanced cervical cancer. Within the past few decades, radiotherapy has evolved and developed significantly in terms of technology, radiobiology and methods improving its effectiveness (irradiation under high pressure oxygen, delivery of radio-sensitizers). During the past few years, combined treatment of irradiation and chemotherapy has been used. This treatment method has been recommended in 1999 by the National Institute of Cancer in the U.S.A. based on the positive results of clinical researches.

Based on the analysis of publications regarding this issue it could be stated that:

1. researches included heterogeneous groups of patients including those treated surgically,

2. patient qualified to treatment were at different advanced stages of disease (from IB to IVA),
3. there exist differences between the numbers of patients at particular advanced stages of disease (with predominance of patients at stage I and II),

4. researches included different cytostatics, delivered according to different treatment schemes,

5. radiotherapy was not conducted according to standardized regulations,

6. patients with different type of cervical cancer participated in researches.

The main goal of presented study was to avoid mentioned above disadvantages and perform analysis on as much as possible homogenous group of patients.

\section{RESEARCH PROBLEM}

The procedure of introduction of every new diagnostic and therapeutic techniques must be always confirmed by long term clinical investigations. These investigations should verify not only the usefulness of the new method but also its supremacy over techniques which are actually in use. Because of high costs and risk for the patient it is desired to find reliable methods which may be based on as small as possible amount of data. As was mentioned earlier radiotherapy is the fundamental treatment method for patients with advanced cervical cancer. The aim of this research was to evaluate the effectiveness of radiochemotherapy in the uniform group of patients with advanced stage of cervical cancer IIIB, differentiation of cancer (squamous epithelial cell carcinoma), and the principals of 
radio-chemotherapy. Until now there has not been many researches performed with such a large number homogenous group of patients (337) with advanced stage IIIB of cervical cancer. Therefore the research may be found original since it does not repeat any other research performed so far [1]. Moreover any other advanced stage of cancer treated with the proposed treatment scheme would lead to completely different results.

In presented research the analysis of prognostic factors and the toxicity of this treatment method were also performed. The main goal was achieved by the analysis of the clinical material obtained during the treatment of 337 patients with squamous epithelial cell carcinoma of cervix at the advanced stage IIIB according to FIGO classification. Patients were divided into two groups: group I patients treated with radiochemotherapy (RCHT) from June 1999 to March 2002; group II - patients treated with radiotherapy alone (RT) within1995 and 1997.

Both groups of patients differed in terms of the performed treatment. 130 patients were treated with the combined treatment of ionizing irradiation and chemotherapy (cisplatin). 207 patients were treated only with radical radiotherapy. In both groups of patients (RCHT and RT) radical radiotherapy consisted of teleradiotherapy and brachytherapy (intracavitary).

\section{DESCRIPTION OF MATHEMATICAL SOLUTION}

The data were imported from original oncological database from Great Poland Cancer Center in Poznań. Before analysis data were precisely examined. Wrong data were either corrected or replaced with symbol of missing data. One hundred and fifty seven variables describing among other things description of the course of teleradiotherapy and/or chemotherapy, personal descriptors were analyzed.

At first step descriptive statistics was calculated (central tendency measures, measure of spreading, frequency tables) depending on measurement scale.

To compare means of parameters in interval scale unpaired $t$-Student test or Welch test was used. In the case of lack of normality Mann-Whitney test was used to check differences between medians. To analyze contingency table $2 \times 2$ or bigger there was used Fisher exact test or Fisher Freeman Halton test. Most of calculations were performed with StatSoft, Inc. (2005) STATISTICA (data analysis software system), version 7.1 (except of Fisher-FreemanHalton test - calculated with Cytel Software Corporation StatXACT-5 release 5.0.3 and Receiver Operating Characteristics analyzed with Analyze-it release 1.71).

The Kaplan-Meier Product-Limit method was used to estimate survival functions $S(t)[2,3]$ :

$$
S(t)=\prod_{j=1}^{t}\left[(n-j) /(n-j+1]^{\delta(j)}\right.
$$

where $\delta(j)$ is a constant that is either 1 if the $j$ '-th case is uncensored or 0 if it is censored, $t-$ is survival time and $n$ is total number of patients. Survival functions were compared either between RT and RCHT treated patients or between patients treated with the same therapeutical method but with differently defined other descriptors (i.e. different total radiation dose). The last type of comparisons allows us to find the influence of individual factor on survival time. To compare two survival functions we used the following tests:

- Wilcoxon test with Gehan correction to evaluate whole survival curves $[3,4]$,

- Wilcoxon test with Peto \& Peto correction to emphasize differences at the beginning of the curves $[3,5]$,

- log-rank test to emphasize differences in final segment of survival curves [3].

Asymptotic chi-square test was used in the case of simultaneous comparison of more than two survival curves.

Problem of finding localization of cut off points for particular predictor measured in interval or ordinal scale was resolved as follows. Series of pairs of survival curves was estimated for the grid consisted of chosen measurement values from the range of analyzed covariate: first curve based on the cases with covariate value below grid point and the second one based on the cases with covariate value above grid point. As a final cut off point we took that grid point (covariate value) for which minimum $p$-value for Gehan test statistics was observed. The described procedure was followed by ROC analysis and finding that one covariate value for which sensitivity and specificity simultaneously reach their maxima. In most of the cases results of both techniques were nearly identical. For predictors measured in nominal scale described grid technique may be only used. Figure 1 presents an example of one factor model (the total dose of radiation on perimetrium). The cutoff point of radiation dose equal to 63 Gy was confirmed both by grid and ROC based methods. For mentioned dose all three survival curves comparison tests (Gehan, Peto-Peto and log-rank) reach highest level of statistical significant difference.

For RT and RCHT groups received survival curves had different length because clinical investigations in both therapeutic groups were conducted in different time. First part of analysis was conducted in the middle of 2003 hence the survival time for RT group spans a period of 8 years while for RCHT group only 4 years. Exponential model was used to standardize length of curves (Fig. 2). The parameters of this model were estimated with quasi-Newton procedure and finally over 93-percentage variance explanation was obtained.

Predictive values resulting from estimated exponential model were compared to real data collected one year after finishing first part of investigation. Statistical Gehan, Peto-Peto and log-rank tests proved that there does not exist significant differences between theoretical (estimated from 
exponential model) and experimental data. Exponential model turned out to be reliable and effective. However one must keep in mind pitfalls which may occur in extrapolation process. In statistical practice as a rule of thumb it is assumed that extrapolation procedure is safe in the region of ten percent of the range of independent variable. In our case it is equivalent to the interval of half a year length. This is the reason why our experiment will be continued at least next four years.

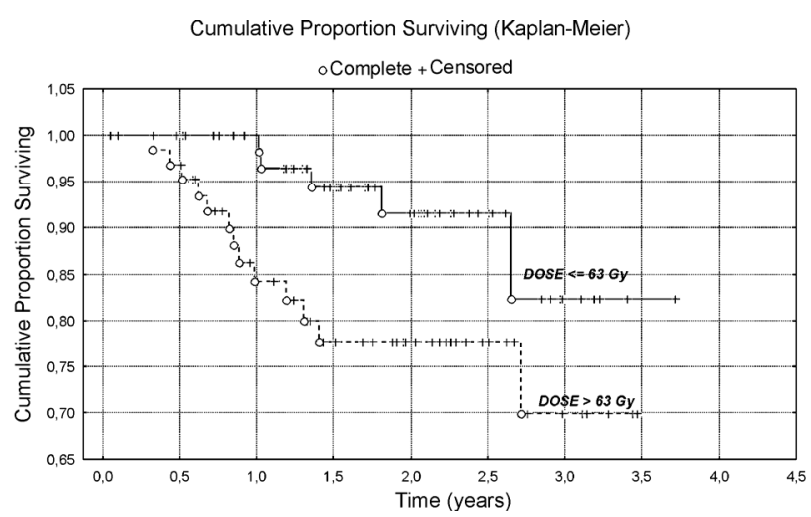

Fig. 1. The influence of total radiation dose on perimetrium on cumulative proportion surviving in radiochemotherapy treated patients (RCHT)

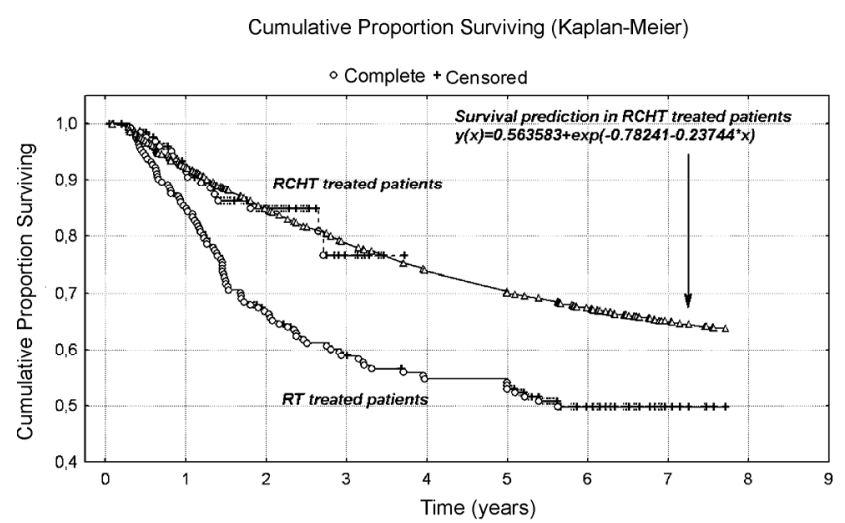

Fig. 2. Cumulative proportion surviving in radiotherapy (RT) and radiochemotherapy (RCHT) treated patients and survival prognosis based on exponential model estimated for RCHT patients group

In the study of the effectiveness of a new treatment for a generally terminal disease the major variable of interest is the number of days that the respective patients survive. On the other hand we are interested in predictors which significantly affect survival function. Both one and multifactor mathematical models were used to determine influence of one or more parameters on survival time. In both cases Cox's Proportional Hazard Model [3, 6] was used:

$$
h\left\{(t),\left(z_{1}, z_{2}, \ldots, z_{m}\right)\right\}=h_{0}(\mathrm{t}) * \exp \left(b_{1} * z_{1}+\ldots+b_{m}{ }^{*} z_{m}\right)
$$

where $h(t, \ldots)$ denotes the estimated hazard function, given the values of the $m$ predictors for the respective case $\left(z_{1}, z_{2}\right.$, $\left.\ldots, z_{m}\right)$ and the respective survival time $(t)$ and $h_{0}(t)$ (called the baseline hazard) is the hazard for the respective individual assuming all independent variable values are equal to zero.

To use that general model only two main assumptions must be fulfilled:

1. there is a log-linear relationship between the independent variables and the underlying hazard function,

2. the ratio of the hazard functions for two observations with different values for the independent variables does not depend on time.

No additional assumptions concerning the nature or shape of the analyzed survival distribution are needed.

There is no simple relation between one and multidimensional models. In other words variables which are significant in one dimensional models may not be the part of multidimensional models. This phenomenon may occur due to predictors redundancy. On the other hand variables which are not significant in one-dimensional models can be significant in construction of multidimensional models. The cause of such a situation can yield also from the correlation between variables [7].

Separate multifactor Cox proportional hazards models were created for both of the treatment groups (RT, RCHT) using results from one factor analysis. As an example in table 1 multidimensional Cox's model parameters for RCHT group are presented. As independent variables we used these five variables which influence time survival in one dimensional models the strongest. Because calculated $p$-value is equal to 0.01669 estimated model is statistically significant and can be used to interpret influence of used variables on survival time. Hazard ratio (exponent beta) and corresponding $p$-value indicate that in RCHT group the strongest influence on time survival have cancer's stage (tumor status) and number of chemotherapy courses. Medical interpretation of all results is discussed in details in Roszak [1].

Table 1. Multidimensional Cox model for the patients treated with RCHT

\begin{tabular}{|c|c|c|c|c|c|c|}
\hline & \multicolumn{6}{|c|}{$\begin{array}{l}\text { Dependent Variable: SURVIVAL TIME } \\
\text { Censoring var.: status } \\
\text { Chi2 }=13,8371 \mathrm{df}=5 p=, 01669 \\
\text { Include condition: } \mathrm{w158=2}\end{array}$} \\
\hline & Beta & $\begin{array}{c}\text { Standard } \\
\text { Error }\end{array}$ & t-value & $\begin{array}{c}\text { exponent } \\
\text { beta }\end{array}$ & $\begin{array}{l}\text { Wald } \\
\text { Statist. }\end{array}$ & $p$ \\
\hline TOTAL DOS & 0,00073 & 0,001474 & 0,49702 & 1,000733 & 0,247026 & \\
\hline PARAMETF & 0,00732 & 0,004595 & 1,59207 & & & \\
\hline LDR_DOSE_A_(left+right) & 0,00011 & 0,001103 & 0,09677 & 1,000107 & 0,009365 & 0,92290 \\
\hline NUMBER O & $-1,31962$ & 0,558365 & $-2,36336$ & 0,267237 & 5,585484 & \\
\hline TUMOR STATUS (G1-G2 - G3) & 0,86551 & 0,373832 & 2,31524 & 2,376216 & 5,360319 & 0,020606 \\
\hline
\end{tabular}

The next step of research was to carry out toxicity analysis of both treatment methods. Beside classical statistical analysis classification and regression trees technique (data mining method) was used [7]. Its flexibility (no need 
to make any assumptions on data distribution, data may be measured in each statistical scale) and interpretability (method mimics the way that a doctor thinks) make that technique very attractive. The reason why classification and regression trees are not popular and rather rarely used by doctors are two main disadvantages:

1. high degree of instability (even small variations in the data can change totally the structure of classification and regression tree, which property yields from hierarchical structure of algorithm),

2. problem how large should one grow the tree (to small tree may not capture the important data structure, to large one may overfit the data).

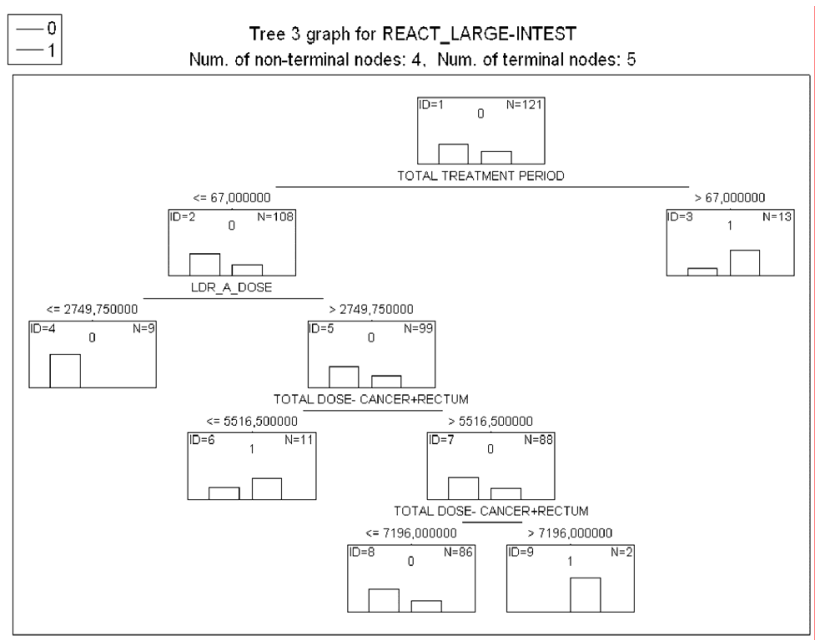

Fig. 3. Classification tree created for explanation of chosen risk factors on occurrence of early large intestine reaction on radiation in RCHT treated patients

Figure 3 presents example of classification tree explaining the influence of particular risk factors on early large intestine reaction on radiation. Eight chosen by doctors risk factors were used as predictors (continuous or categorical), categorical response (dependent variable) was described

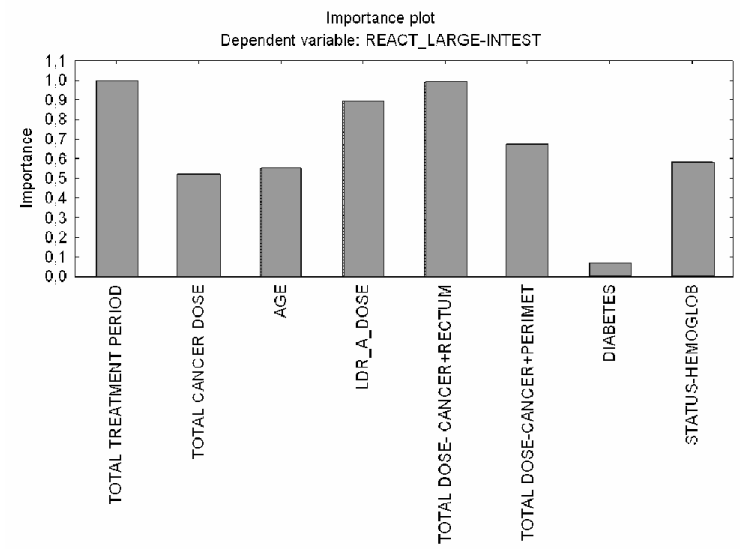

Fig. 4. Importance plot for predictors used to create tree classifier presented in Fig. 3 either by 0 (lack of early large intestine reaction) or 1 (early large intestine reaction exists). Goodness of fit was estimated with Gini measure (measure of node impurity), prior probabilities were estimated on the basis of subgroup sizes, loss matrix was symmetrical, prune on misclassification error was used as a stopping rule. The relative importance of particular variables in prediction of reaction is shown on Fig. 4. It is easy to find that all predictors directly related to radiation dose play the dominant role in comparison to other factors such as age, existence of diabetes, status of hemoglobine level. The only one exception is total treatment period.

Resubstitution cost estimate is the proportion of cases that are misclassified by the tree constructed from the entire sample. It is computed using the same data as used in constructing the tree classifier. From Table 2 we can state, that resubstitution cost for early large intestine reaction is equal to $28.93 \%$ (35 misclassified cases). It means that using presented tree we are able to explain the dependence between analyzed predictors and intestine reaction correctly in over seventy percents with all the cases we used to create that tree. But it is not the same as prediction of intestine reaction using values of predictors for new (unknown for tree classifier) patients. To estimate the quality of classifier we must use either additional test data (data which were not used to construct the tree) or perform crossvalidation procedure. Because of rather small sample size we decided to use this last technique. After ten-fold cross-validation we obtain for analyzed tree $\mathrm{CV}$ error equal to $0.504132 \pm 0.045453$. Final conclusion is that however presented tree properly explains the structure of existing experimental data it should not be used for prediction of outcomes for new unknown cases.

Table 2. Classification matrix based on results obtained with tree presented on Fig. 3

\begin{tabular}{|c|c|c|c|c|c|}
\hline & \multicolumn{5}{|c|}{$\begin{array}{l}\text { Classification matrix } 3 \text { (DANE28072003-MODIFIED-101.STA) } \\
\text { Dependent variable: REACT_LARGE-INTEST } \\
\text { Options: Categorical response, Analysis sample } \\
\text { Include condition: } v 158=2\end{array}$} \\
\hline & Observed & Predicted 0 & \begin{tabular}{|l|} 
Predicted 1 \\
\end{tabular} & Row Total & \\
\hline Number & [ & 67 & 7 & 74 & \\
\hline Column Percentage & & $70.53 \%$ & $26.92 \%$ & & \\
\hline Row Percentage & & $90.54 \%$ & $9.46 \%$ & & \\
\hline Total Percentage & & $55.37 \%$ & $5.79 \%$ & $61.16 \%$ & \\
\hline Number & 1 & 28 & 19 & 47 & \\
\hline Column Percentage & & $29.47 \%$ & $73.08 \%$ & & \\
\hline Row Percentage & & $59.57 \%$ & $40.43 \%$ & & \\
\hline Total Percentage & & $23.14 \%$ & $15.70 \%$ & $38.84 \%$ & \\
\hline Count & All Groups & 95 & 26 & $\overline{121}$ & \\
\hline Total Percent & & $78.51 \%$ & $21.49 \%$ & & \\
\hline
\end{tabular}

When we analyze the structure of tree presented on Fig. 3, we can state that split values generated automatically according to algorithm are not simple and doctors may have problems with remembering such generated "if-then" logical rules. To avoid this disadvantage one can use interactive C\&RT trees and change split values manually to the nearest logical and easy to remember one. 


\section{CONCLUSIONS}

Statistical analysis and collected data of female with squamous epithelial cell carcinoma of cervix at the advanced stage IIIB let us to draw the following conclusions [1]:

1. Treating female with squamous epithelial cell carcinoma of cervix at the advanced stage IIIB better is to use radio chemotherapy than radiotherapy $(p=0.001)$.

2. There exist differences in independent predictive factors:

- number of chemotherapy $(p=0.043)$ and stage of the cancer $(p=0.01)$ in group which was treated radio-chemotherapy,

- hemoglobin's level during course of treatment $(p=$ $0.04)$, age $(p=0.04)$, dose of ionizing radiation $(p=0.018)$ in group which was treated brachytherapy.

3. In both of treatment's methods there was no escalating of radiation sickness in healthy tissues.

4. The radio-chemotherapy caused bigger hemolytic toxicity in hemoglobin's level $(p=0.000)$, count of leucocytes $(p=0.000)$ and granulocytes $(p=0.000)$ than radiotherapy. Escalating of toxicity wasn't interfered with treatment.

5. The radio-chemotherapy is better method of treatment female with squamous epithelial cell carcinoma of cervix at the advanced stage IIIB because of greater effectiveness, not too big hemolytic toxicity, and lack of differences in incidence radiation sickness in healthy tissues (compare to radiotherapy).

6. Choice of predictive factors significantly differs for patients with squamous epithelial cell carcinoma of cervix which had radiotherapy and patients with squamous epithelial cell carcinoma of cervix which had radio-chemotherapy.

\section{References}

[1] A. Roszak, Badania nad skutecznościa radiochemioterapii chorych na zaawansowanego raka szyjki macicy, Rep. Pract. Oncol. Radiother. 9 (S1), 131-162 (2004).

[2] E. L. Kaplan and P. Meier, Nonparametric estimation from incomplete observations, Journal of the American Statistical Association, 53, 457-481 (1958).

[3] Encyclopedia of Biostatistics, ed. P. Armitage, T. Colton, J. Wiley \& Sons, Ltd. (2005).

[4] E. A. Gehan, A generalized Wilcoxon test for comparing arbitrarily singly-censored samples, Biometrika, 52, 203-223 (1965a).

[5] R. Peto and J. Peto, Asymptotically efficient rank invariant procedures, Journal of the Royal Statistical Society, 135, 185-207 (1972).

[6] D. R. Cox and D. Oakes, Analysis of survival data, New York: Chapman \& Hall (1984).

[7] T. Hastie, R. Tibshirani and J. Friedman, The elements of Statistical Learning. Data Mining, Inference and Prediction, Springer-Verlag NY (2001).

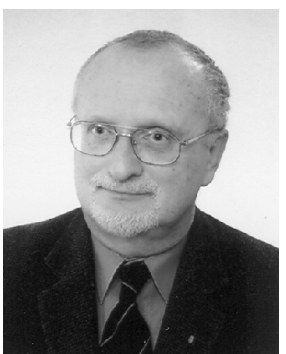

Jerzy A. Moczko was born in Poznań, Poland, in 1951. He received his MSc degree in theoretical physics in 1973 from the Jagiellonian University in Cracow. He earned the PhD in biophysics in 1982 from Poznań University of Medical Sciences in Poznan. He habilitated in 1990 and since 1998 he is the Full Professor and Head of the Chair and Department of Computer Science and Statistics at Poznań University of Medical Sciences in Poznań. His research interests concern, amongs other topics, methods of digital processing of biomedical signals and methods of medical diagnosis support based on statistical, artificial intelligence and data mining techniques. 